\title{
Relationship between Weather Parameters and Sunflower Powdery Mildew (Erysiphe cichoracearum DC) in Various Sunflower Sowing Dates
}

\author{
A. Bheemaraya*, M.M. Jamadar and Shalini Huilgol
}

Department of Plant Pathology, College of Agriculture, Bijapur, University of Agricultural Sciences, Dharwad-580 005, Karnataka, India

*Corresponding author

\begin{tabular}{|c|}
\hline Keywords \\
\hline $\begin{array}{l}\text { Dates of sowing, } \\
\text { Powdery mildew } \\
\text { (Erysiphe } \\
\text { cichoracearum), Weather } \\
\text { parameters }\end{array}$ \\
\hline Article Info \\
\hline $\begin{array}{l}\text { Accepted: } \\
24 \text { August } 2018 \\
\text { Available Online: } \\
\text { 10 September } 2018\end{array}$ \\
\hline
\end{tabular}

\section{Introduction}

Sunflower (Helianthus annuus L.) is native of southern USA and Mexico. It is an important oilseed crop belonging to Asteraceae family and ranks third next to groundnut and

\section{A B S T R A C T}

A study was conducted in Karnataka, India from 2013-14 at College of Agriculture, Bijapur, (UAS, Dharwad) to know the effect of weather parameters in seven dates of sowing from I Fortnight (FN) July to I FN of October on sunflower powdery mildew. Weather correlations in I FN July dates shown significant positive impact of cloud cover at 20-1 days before observation (DBO) on the powdery mildew incidence, highly significant parameters that affected the powdery mildew at 20-16 DBO were minimum temperature ($0.95)$ as well as maximum temperature $(0.98)$ and maximum vapour pressure at $(-0.95)$ at 5-1 DBO. The weather correlations in II FN July date of sowing indicated highly significant negative relation of minimum temperature (-0.88) at 20-1 DBO, At 5-1 DBO evening relative humidity $(0.81)$ showed positive relationship. Correlations of weather parameters in I FN August sowing date indicated that at 20-1 DBO all the weather showed negative and highly significant relationship except cloud cover $(0.83)$ which was positive and significantly correlated. Interestingly rainfall $(-0.84)$ indicated negatively significant relationship with powdery mildew severity. The weather correlations in II FN August sowing indicated that at 20-1 DBO highly significant negative relationship with most of weather parameters but rain fall (-0.79) significant and negative at 20-1 DBO. Crop sown in I FN of September revealed At 20-16 DBO none of the weather parameters had the significant influence on the powdery mildew intensity except maximum temperature ($0.83)$ which was significantly negatively correlated, here rainfall $(-0.85)$ was significant and negatively affected the powdery mildew severity. In II FN September sowing indicated that highly significant and negative relation of maximum temperature $(-0.95)$ among all the weather variables on powdery mildew intensity at 5-1 DBO. Powdery mildew severity in I FN October sown crop were observed except morning relative humidity (-0.78) at 20-1 DBO and maximum temperature (0.88) at 20-16 DBO.

soybean. It is a rich source of edible oil (40$52 \%$ ) having anticholesterol properties due to the presence of poly unsaturated fatty acids (55-65\% linoleic acid and 20-30\% oleic acid) which are known to reduce the risk of coronary diseases (Joksimovic et al., 2006). 
Although India is the third largest producer of the oilseeds, the country is facing an acute shortage of edible oil. Presently in India sunflower is cultivated over an area of 1.72 mha with a production of 0.50 MT and productivity of $692 \mathrm{~kg} / \mathrm{ha}$ (Anon., 2013). The cultivated sunflower is largely confined to southern parts of the country comprising the states like Andhra Pradesh, Karnataka, Maharashtra and Tamil Nadu. Karnataka stands first among these southern states accounting for an area of 0.38 mha with a production of 0.19 MT and productivity of $503 \mathrm{~kg} / \mathrm{ha}$; thus contributing for a major share $(42.83 \%)$ in the country's sunflower production (Anon., 2012). Sunflower is photo insensitive and thus it can be grown round the year (Reddy, 2012).

The lower levels of sunflower yields are mainly due to several biotic and abiotic factors. Among these, susceptibility to disease is considered to be one of the major constraints. Kolte (1985) observed that the crop suffers from various diseases incited by fungi, bacteria, viral and phytoplasma. Among the fungal disease of sunflower Alternaria leaf spot (Alternaria helianthi), rust (Puccinia helianthi), downy mildew (Plasmapara halstedii Farl.), are important. Recently, powdery mildew and necrosis disease have become most important limiting factors of cultivation in sunflower. Powdery mildew like other fungal diseases is influenced by weather factors. Generally, it prevails in dry conditions, which is commonly experienced in the northern dry zone of Karnataka therefore influence of weather parameters such as temperature, relative humidity $(\mathrm{RH})$ and other microclimatic factors is very important to develop forewarning system in this disease. Keeping these facts, the investigation was carried out to know the Effect of weather parameters on sunflower powdery mildew (Erysiphe cichoracearum DC) in various sunflower sowing dates.

\section{Materials and Methods}

The experiment was conducted in research fields of College of Agriculture, Bijapur in a Randomized Complete Block Design (RCBD) with three replications and seven dates of sowings (I Fortnight of July, II Fortnight of July, I Fortnight of August, II Fortnight of August, I Fortnight of September, II Fortnight of September and I Fortnight of October). Individual plot size was $5 \times 4 \mathrm{~m}^{2}$. Sunflower seeds were sown at $60 \mathrm{~cm}$ spacing between rows and $30 \mathrm{~cm}$ between plants. The first date of sowing was imposed by sowing seeds of highly susceptible hybrid 'KBSH-44' in 1st fortnight of July and subsequent sowings were done at an interval of 15 days till the last sowing date i.e. 1st fortnight of October. Totally seven different date of sowings were imposed in the experiment. The severity of powdery mildew was recorded periodically at 20 days interval after the disease appearance on five randomly selected plants per plot using 0-5 disease rating scale and Per cent Disease Index (PDI) were worked out. The weather data (experimental period) of RARS, Bijapur was collected from meteorological unit and used for correlation with the powdery mildew severity.

Further, per cent disease index (PDI) was calculated by using following formula proposed by Wheeler (1969).

Per cent disease index $=\frac{\text { Sum of the individual disease ratings }}{\text { Number of leaves assesed } X \text { Maximum grade }} \times 100$

\section{Results and Discussion}

Effect of date of sowing on sunflower powdery mildew (Erysiphe cichoracearum DC) disease severity

The various dates of sowing of sunflower on powdery mildew severity indicated that sowing of crop in the II fortnight (FN) of July 
recorded the minimum powdery mildew infection $(8.80 \%)$ followed by crop sown in I FN of August $(8.82 \%)$ as against the crop sown in I FN of October (39.64\%) and II FN of September (27.18\%). It was also observed that the disease progressed linearly from initial disease incidence $(4.08 \%)$ recorded at 40DAS and reached the highest disease intensity $(37.92 \%)$ at 100 DAS. However there was exception to this disease progress in the early sowing dates that indicated the decline in the disease development at $80 \mathrm{DAS}$ as evident from July I FN (7.70\%) and II FN (5.05\%) as well as sowing in the August I FN (2.76\%) sowing recorded low powdery mildew after fairly higher disease intensity at 60 DAS. At 100 DAS the disease intensity again increased indicating the definite as well as significant meteorological interventions (Table 1).

\section{Relationship of weather parameters with sunflower powdery mildew in individual sowing dates}

Further the weather correlations with individual dates of sowing (Table 2) revealed significant positive impact of cloud cover at 20-1 DBO on the powdery mildew incidence of the crop sown in I FN July. While highly significant parameters that affected the powdery mildew at 20-16 DBO were minimum temperature (-0.95) as well as maximum temperature $(0.98)$ and maximum vapour pressure at (-0.95) at 5-1 DBO. It was also observed that significant and positive impact ( 0.85 each) of cloud cover on powdery mildew intensity at 20-16 and 20-1 DBO, respectively.

The weather correlations in II FN July date of sowing (Table 3) revealed highly significant negative relation of minimum temperature (0.88 ) at $20-1$ DBO on the powdery mildew severity, while significant relationship of maximum vapour pressure $(0.78)$ that affected the powdery mildew at 20-16 DBO. At 5-1
DBO maximum temperature (-0.81) indicated significant and negative impact with powdery mildew whereas evening relative humidity (0.81) showed positive relationship.

Correlations of weather parameters of crop sown in I FN August (Table 4) revealed that at 20-1 DBO all the weather parameters like maximum temperature (-0.91), minimum temperature (-0.95), maximum vapour pressure (-0.96), minimum vapour pressure (0.92 ), morning and evening relative humidity (-0.94 each) showed negative and highly significant relationship except cloud cover $(0.83)$ which was positive and significantly correlated. Interestingly rainfall (-0.84) indicated negatively significant relationship with powdery mildew severity. At 20-16 DBO minimum temperature $(-0.89)$ and maximum vapour pressure (-0.87), minimum vapour pressure $(-0.90)$ as well as morning relative humidity (-0.96) indicated significant to highly significant and negative impact on powdery mildew incidence. Here too the rainfall $(-0.93)$ had highly significant and negative relationship. At 5-1 DBO negative and highly significant relationship of minimum temperature (-0.94), maximum vapour pressure (-0.97), minimum vapour pressure (-0.99), morning relative humidity ($0.99)$ and evening relative humidity $(-0.95)$ was observed, in case of cloud cover (0.77) positively significant relationship was observed but maximum temperature and rainfall has shown no significant relationship on powdery mildew disease severity at this crop growth stage. The weather correlations with powdery mildew severity in II FN August sowing (Table 5) revealed that at 20-1 DBO highly significant negative relationship of maximum temperature (-0.87), minimum temperature (-0.99), maximum vapour pressure $(-0.96)$, minimum vapour pressure ($0.93)$, and morning relative humidity $(-0.79)$ was observed while cloud cover (0.89) was highly significant and positive. 
The weather data (experimental period) of RARS, Bijapur

\begin{tabular}{|c|l|}
\hline Disease rating & Percent incidence/ leaf infection \\
\hline 0 & No disease \\
\hline 1 & $1-10 \%$ infection \\
\hline 2 & $11-25 \%$ infection \\
\hline 3 & $26-50 \%$ infection \\
\hline 4 & $51-75 \%$ infection \\
\hline 5 & $>75 \%$ infection \\
\hline
\end{tabular}

Table.1 Effect of dates of sowing of sunflower on powdery mildew severity and yield

\begin{tabular}{|c|c|c|c|c|c|}
\hline \multirow{3}{*}{ Treatments } & \multicolumn{5}{|c|}{ Per cent Disease Index (PDI) } \\
\hline & \multicolumn{4}{|c|}{ Days after sowing (DAS) } & \multirow[t]{2}{*}{ Mean } \\
\hline & 40 & 60 & 80 & 100 & \\
\hline I Fortnight of July & $\begin{array}{c}0.79 \\
(5.10)^{*}\end{array}$ & $\begin{array}{c}13.84 \\
(21.84)\end{array}$ & $\begin{array}{c}7.70 \\
(16.11)\end{array}$ & $\begin{array}{c}27.11 \\
(31.38)\end{array}$ & $\begin{array}{c}12.36 \\
(20.58)\end{array}$ \\
\hline II Fortnight of July & $\begin{array}{c}0.66 \\
(4.67)\end{array}$ & $\begin{array}{c}9.95 \\
(18.39)\end{array}$ & $\begin{array}{c}5.05 \\
(12.99)\end{array}$ & $\begin{array}{c}19.52 \\
(26.22)\end{array}$ & $\begin{array}{c}8.80 \\
(17.26)\end{array}$ \\
\hline I Fortnight of August & $\begin{array}{c}1.53 \\
(7.10)\end{array}$ & $\begin{array}{c}8.60 \\
(17.05)\end{array}$ & $\begin{array}{c}2.76 \\
(9.56)\end{array}$ & $\begin{array}{c}22.37 \\
(28.23)\end{array}$ & $\begin{array}{c}8.82 \\
(17.28)\end{array}$ \\
\hline II Fortnight of August & $\begin{array}{c}2.60 \\
(9.28)\end{array}$ & $\begin{array}{c}2.62 \\
(9.32)\end{array}$ & $\begin{array}{c}22.67 \\
(28.44)\end{array}$ & $\begin{array}{c}36.16 \\
(36.97)\end{array}$ & $\begin{array}{c}16.01 \\
(23.59)\end{array}$ \\
\hline I Fortnight of September & $\begin{array}{c}2.55 \\
(9.19)\end{array}$ & $\begin{array}{c}15.61 \\
(23.27)\end{array}$ & $\begin{array}{c}17.95 \\
(25.07)\end{array}$ & $\begin{array}{c}28.50 \\
(32.27)\end{array}$ & $\begin{array}{c}16.15 \\
(23.70)\end{array}$ \\
\hline II Fortnight of September & $\begin{array}{c}4.04 \\
(11.59)\end{array}$ & $\begin{array}{c}7.75 \\
(16.17)\end{array}$ & $\begin{array}{c}36.59 \\
(37.22)\end{array}$ & $\begin{array}{c}60.35 \\
(50.97)\end{array}$ & $\begin{array}{c}27.18 \\
(31.42)\end{array}$ \\
\hline I Fortnight of October & $\begin{array}{c}16.40 \\
(23.89)\end{array}$ & $\begin{array}{c}23.00 \\
(28.66)\end{array}$ & $\begin{array}{c}47.67 \\
(43.67)\end{array}$ & $\begin{array}{c}71.48 \\
(57.72)\end{array}$ & $\begin{array}{c}39.64 \\
(39.02)\end{array}$ \\
\hline S. Em \pm & 1.00 & 2.06 & 1.94 & 1.97 & \\
\hline C.D. $(P=0.05)$ & 3.10 & 6.36 & 6.00 & 6.09 & \\
\hline
\end{tabular}

* Figures in the parentheses are arc sine transformed value

Table. 2 Correlation coefficient between weather parameters and powdery mildew of sunflower sown during I FN July

\begin{tabular}{|c|c|c|c|c|}
\hline \multicolumn{2}{|c|}{ Per cent disease index (PDI) } & \multicolumn{2}{|c|}{12.36} & \multirow{3}{*}{$\begin{array}{c}\text { 20-1 DBO } \\
0.74\end{array}$} \\
\hline \multicolumn{2}{|c|}{ Weather parameters } & 5-1 DBO & 20-16 DBO & \\
\hline \multirow[t]{2}{*}{ Temperature $\left({ }^{\circ} \mathrm{C}\right)$} & Max. & 0.47 & $0.98 * *$ & \\
\hline & Min. & $-0.75^{*}$ & $-0.95 * *$ & $-0.76^{*}$ \\
\hline \multirow{2}{*}{ Vapour pressure (\%) } & Max. & $-0.95 * *$ & -0.60 & -0.28 \\
\hline & Min. & -0.32 & -0.62 & -0.37 \\
\hline \multirow[t]{2}{*}{ Relative humidity (\%) } & Morn. & 0.07 & 0.13 & -0.06 \\
\hline & Even. & -0.35 & $-0.79 *$ & -0.60 \\
\hline \multicolumn{2}{|l|}{ Cloud cover } & 0.45 & $0.85^{*}$ & $0.85 *$ \\
\hline \multicolumn{2}{|l|}{ Rainfall (mm) } & 0.02 & -0.30 & -0.42 \\
\hline
\end{tabular}

DBO - Day Before Observation

* Significant at 5\%,** Significant at $1 \%$ 
Table.3 Correlation coefficient between weather parameters and powdery mildew of sunflower sown during II FN July

\begin{tabular}{|c|c|c|c|c|}
\hline Per cent disease indes & \multicolumn{4}{|c|}{8.80} \\
\hline \multicolumn{2}{|c|}{$\begin{array}{l}\text { Weather parameters } \\
\end{array}$} & 5-1 DBO & 20-16 DBO & 20-1 DBO \\
\hline \multirow{2}{*}{ Temperature $\left({ }^{\circ} \mathrm{C}\right)$} & Max. & $-0.81 *$ & 0.30 & $-0.88 * *$ \\
\hline & Min. & -0.72 & 0.20 & -0.52 \\
\hline \multirow[t]{2}{*}{ Vapour pressure (\%) } & Max. & -0.61 & $0.78 *$ & -0.30 \\
\hline & Min. & -0.29 & 0.01 & -0.11 \\
\hline \multirow[t]{2}{*}{ Relative humidity (\%) } & Morn. & -0.44 & 0.09 & -0.37 \\
\hline & Even. & $0.81 *$ & -0.31 & 0.33 \\
\hline \multicolumn{2}{|l|}{ Cloud cover } & 0.11 & 0.42 & 0.19 \\
\hline \multicolumn{2}{|l|}{ Rainfall (mm) } & 0.07 & 0.06 & 0.45 \\
\hline
\end{tabular}

DBO - Day Before Observation

* Significant at 5\%,** Significant at $1 \%$

Table.4 Correlation coefficient between weather parameters and powdery mildew of sunflower sown during I FN August

\begin{tabular}{|c|c|c|c|c|}
\hline Per cent disease index & \multicolumn{4}{|c|}{8.82} \\
\hline \multicolumn{2}{|c|}{ Weather parameters } & 5-1 DBO & 20-16 DBO & 20-1 DBO \\
\hline \multirow[t]{2}{*}{ Temperature $\left({ }^{\circ} \mathrm{C}\right)$} & Max. & -0.37 & -0.71 & $-0.91 * *$ \\
\hline & Min. & $-0.94 * *$ & $-0.89 *$ & $-0.95 * *$ \\
\hline \multirow[t]{2}{*}{ Vapour pressure (\%) } & Max. & $-0.97 * *$ & $-0.87 *$ & $-0.96 * *$ \\
\hline & Min. & $-0.99 * *$ & $-0.90 * *$ & $-0.92 * *$ \\
\hline \multirow[t]{2}{*}{ Relative humidity (\%) } & Morn. & $-0.99 * *$ & $-0.96 * *$ & $-0.94 * *$ \\
\hline & Even. & $-0.95 * *$ & -0.14 & $-0.94 * *$ \\
\hline \multicolumn{2}{|l|}{ Cloud cover } & $0.77 *$ & 0.54 & $0.83 *$ \\
\hline \multicolumn{2}{|l|}{ Rainfall (mm) } & -0.67 & $-0.93 * *$ & $-0.84 *$ \\
\hline
\end{tabular}

DBO - Day Before Observation

* Significant at 5\%,** Significant at $1 \%$

Table.5 Correlation coefficient between weather parameters and powdery mildew of sunflower sown during II FN August

\begin{tabular}{|c|c|c|c|c|}
\hline Per cent disease inde & \multicolumn{4}{|c|}{16.01} \\
\hline \multicolumn{2}{|c|}{$\begin{array}{r}\text { Weather parameters } \\
\end{array}$} & 5-1 DBO & 20-16 DBO & 20-1 DBO \\
\hline \multirow[t]{2}{*}{ Temperature $\left({ }^{\circ} \mathrm{C}\right)$} & Max. & $-0.90 * *$ & $-0.92 * *$ & $-0.87 * *$ \\
\hline & Min. & $-0.77 *$ & $-0.84^{*}$ & $-0.99 * *$ \\
\hline \multirow[t]{2}{*}{ Vapour pressure (\%) } & Max. & $-0.75^{*}$ & $-0.79 *$ & $-0.96 * *$ \\
\hline & Min. & -0.29 & $-0.80 *$ & $-0.93 * *$ \\
\hline \multirow[t]{2}{*}{ Relative humidity (\%) } & Morn. & 0.12 & -0.59 & $-0.79 *$ \\
\hline & Even. & 0.12 & -0.49 & -0.70 \\
\hline \multicolumn{2}{|l|}{ Cloud cover } & -0.20 & 0.47 & $0.89 * *$ \\
\hline \multicolumn{2}{|l|}{ Rainfall (mm) } & $-0.78 *$ & -0.52 & $-0.79 *$ \\
\hline
\end{tabular}

DBO - Day Before Observation

* Significant at 5\%, ** Significant at $1 \%$ 
Table.6 Correlation coefficient between weather parameters and powdery mildew of sunflower sown during I FN September

\begin{tabular}{|c|c|c|c|c|}
\hline Per cent disease index & \multicolumn{4}{|c|}{16.15} \\
\hline \multicolumn{2}{|c|}{$\begin{array}{c}\text { Weather parameters } \\
\end{array}$} & 5-1 DBO & 20-16 DBO & 20-1 DBO \\
\hline \multirow{2}{*}{ Temperature $\left({ }^{\circ} \mathrm{C}\right)$} & Max. & -0.72 & $-0.83 *$ & $-0.96 * *$ \\
\hline & Min. & $-0.95 * *$ & -0.44 & $-0.92 * *$ \\
\hline \multirow[t]{2}{*}{ Vapour pressure (\%) } & Max. & $-0.95 * *$ & -0.35 & $-0.89 * *$ \\
\hline & Min. & $-0.86^{*}$ & -0.38 & $-0.88 * *$ \\
\hline \multirow[t]{2}{*}{ Relative humidity (\%) } & Morn. & $-0.86^{*}$ & 0.51 & $-0.87 * *$ \\
\hline & Even. & -0.70 & -0.32 & $-0.77 *$ \\
\hline \multicolumn{2}{|l|}{ Cloud cover } & 0.47 & 0.13 & $0.77 *$ \\
\hline \multicolumn{2}{|l|}{ Rainfall (mm) } & $-0.85^{*}$ & -0.07 & -0.56 \\
\hline
\end{tabular}

DBO - Day Before Observation

$*$ Significant at 5\%,** Significant at $1 \%$

Table.7 Correlation coefficient between weather parameters and powdery mildew of sunflower sown during II FN September

\begin{tabular}{l|ll} 
Per cent disease index (PDI) & 27.18
\end{tabular}

\begin{tabular}{|l|c|c|c|c|}
\hline \multicolumn{1}{|c|}{ Weather parameters } & & $\mathbf{5 - 1}$ DBO & $\mathbf{2 0 - 1 6}$ DBO & $\mathbf{2 0 - 1}$ DBO \\
\hline Temperature $\left({ }^{\circ} \mathrm{C}\right)$ & Max. & $-0.95^{* *}$ & -0.31 & -0.63 \\
\hline & Min. & -0.56 & $-0.91^{* *}$ & $-0.84^{*}$ \\
\hline Vapour pressure $(\%)$ & Max. & -0.47 & $-0.94^{* *}$ & $-0.80^{*}$ \\
\hline Relative humidity $(\%)$ & Min. & -0.40 & $0.95^{* *}$ & $-0.74^{*}$ \\
\hline Cloud cover & Morn. & 0.52 & $-0.97 * *$ & $-0.75^{*}$ \\
\hline Rainfall $(\mathbf{m m})$ & Even. & -0.15 & $-0.98^{* *}$ & -0.73 \\
\hline
\end{tabular}

DBO - Day Before Observation

* Significant at 5\%,** Significant at $1 \%$

Table.8 Correlation coefficient between weather parameters and powdery mildew of sunflower sown during I FN October

\begin{tabular}{|l|c|c|c|c|}
\hline \multicolumn{1}{|c|}{ Per cent disease index (PDI) } & \multicolumn{4}{|c|}{$\mathbf{3 9 . 6 4}$} \\
\hline \multicolumn{1}{|c|}{ Weather parameters } & & $\mathbf{5 - 1}$ DBO & $\mathbf{2 0 - 1 6 ~ D B O}$ & $\mathbf{2 0 - 1}$ DBO \\
\hline Temperature $\left({ }^{\circ} \mathrm{C}\right)$ & Max. & $0.77^{*}$ & $0.88^{*}$ & 0.46 \\
\hline & Min. & 0.65 & -0.48 & -0.38 \\
\hline Vapour pressure (\%) & Max. & $0.88^{* *}$ & -0.47 & -0.64 \\
\hline Relative humidity (\%) & Min. & 0.30 & -0.47 & -0.72 \\
\hline Cloud cover & Morn. & -0.50 & 0.08 & -0.72 \\
\hline Rainfall $(\mathbf{m m})$ & Even. & 0.50 & -0.30 & $-0.78^{*}$ \\
\hline
\end{tabular}

DBO - Day Before Observation

* Significant at 5\%, ** Significant at 1\% 
In case of rain fall (-0.79) significant and negative relationship with powdery mildew was observed at 20-1 DBO. While highly significant parameters that influence the powdery mildew at 20-16 DBO were maximum temperature (-0.92), whereas significant and negative impact of minimum temperature (-0.84), maximum vapour pressure (-0.79), minimum vapour pressure ($0.80)$, on powdery mildew was noticed at this stage. In case of maximum temperature (0.90) had negative and highly significant correlation, minimum temperature $(-0.77)$ and maximum vapour pressure $(-0.75)$ were significantly negative at 5-1 DBO antecedent weather. In this case too; rainfall $(-0.78)$ had significant negative influence on the powdery mildew intensity.

The results (Table 6) on correlations of antecedent weather parameters (20-1 DBO) with powdery mildew severity on crop sown in I FN of September revealed highly significant and negative correlation with maximum temperature (-0.96), minimum temperature (-0.92), maximum and minimum vapour pressure $(-0.89,-0.88$ respectively) and morning relative humidity $(-0.87)$, while evening relative humidity $(-0.77)$ was negative and significant whereas positive correlation with cloud cover (0.77) was observed. At 20-16 DBO none of the weather parameters had the significant influence on the powdery mildew intensity except maximum temperature $(-0.83)$ which was significantly negatively correlated.

At 5-1 DBO negative, highly significant correlation of minimum temperature $(-0.95)$ and maximum vapour pressure (-0.95) were observed while minimum vapour pressure and morning relative humidity (-0.86 each) indicated negative and significant impact on powdery mildew severity. Here rainfall (0.85) was significant and negatively affected the powdery mildew severity.
The weather correlations with powdery mildew intensity on II FN September sowing (Table 7) revealed significant negative impact of minimum temperature (-0.84), maximum vapour pressure (-0.80), minimum vapour pressure (-0.74) and morning relative humidity $(-0.75)$ at $20-1 \mathrm{DBO}$, in this case cloud cover (-0.97) recorded highly significant and negative relationship. At 2016 DBO weather parameters, minimum temperature (-0.91), maximum vapour pressure (-0.94), morning relative humidity ($0.97)$ and evening relative humidity (-0.98) indicated highly significant and negative relationship whereas cloud cover (0.96) and minimum vapour pressure (0.95) showed highly significant and positive correlation. It was observed that at 5-1 DBO highly significant and negative relation of maximum temperature (-0.95) among all the weather variables with powdery mildew intensity at 51 DBO antecedent weather variables.

There were no significant weather parameters influencing the powdery mildew severity in I FN October sown crop (Table 8) were observed except morning relative humidity (0.78 ) at 20-1 DBO and maximum temperature (0.88) at 20-16 DBO. While positively significant correlation of maximum temperature (0.77) and highly significant relationship maximum vapour pressure $(0.88)$ was observed at 5-1 DBO.

\section{References}

Anonymous, 2012, Directorate of economics and statistics, Department of Agriculture and Co-operation, New Delhi, pp. 107.

Anonymous, 2013, Food and agricultural organisation statics. http://faostat3. fao.org/faostatgateway/go/to/download/ $\mathrm{Q} / \mathrm{QC} / \mathrm{E}$.

Joksimovic, J., Atlagic Jovanka, Marinkovic, R. and Jovanovi, D., 2006, Genetic 
control of oleic and linoleic acid contents in sunflower. Helia, 29(44): $33-40$.

Kolte, S.J., 1985, Diseases of Annual Edible Oilseed Crops III. CRC Press, Florida, pp. 9-96.
Reddy, S.R., 2012, Agronomy of Field Crops, Kalyani Publishers. pp. 415-433. Wheeler, B.E.J., 1969, An Introduction to Plant Disease. John Wiley \& Sons Ltd., London, p. 301.

\section{How to cite this article:}

Bheemaraya, A., M.M. Jamadar and Shalini Huilgol. 2018. Relationship between Weather Parameters and Sunflower Powdery Mildew (Erysiphe cichoracearum DC) in Various Sunflower Sowing Dates. Int.J.Curr.Microbiol.App.Sci. 7(09): 3495-3502.

doi: https://doi.org/10.20546/ijcmas.2018.709.433 